\title{
Multivariate extension of the singular spectrum analysis a new tool in understanding the intraseasonal-oscillation (ISO) of Philippines summer monsoon and its association with extreme rainfall events
}

\author{
Luzano, W.* \\ Department of Applied Mathematics, University of Science \& Technology of Southern Philippines, Philippines \\ Received: 16/08/2019, Accepted: 13/09/2020, Available online: 22/10/2020 \\ *to whom all correspondence should be addressed: e-mail: \\ https://doi.org/10.30955/gnj.003202
}

\section{Graphical abstract}

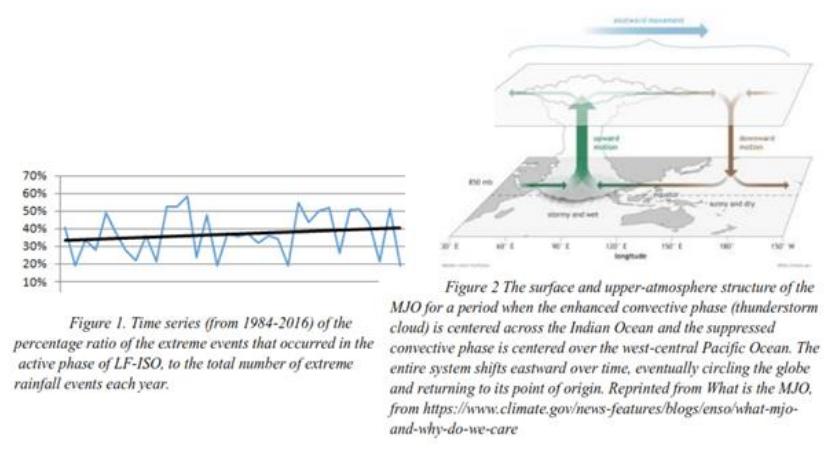

\begin{abstract}
The intraseasonal low-frequency oscillation is studied by using gridded rainfall dataset from 1979 to present. Multivariate Singular Spectrum Analysis (SSA) was used in the extraction of these modes. We have found out that there is a significant increasing trend in the relative strength of low frequency ISO. However, this happened only after the years 1979 to 1984 was removed in the trend analysis. A previous study found that the increased rainfall during the break and transition phase of low-frequency ISO could be a contributing factor to the decreasing trend during the active phase. In this study, the implication is rather the opposite - the increasing trend in rainfall, in terms of extreme events occurrence during the active phase of low-frequency ISO had been the contributor to the increasing trend of low-frequency ISO.
\end{abstract}

Keywords: Singular spectrum analysis, multivariate SSA, intraseasonal oscillations, Madden-Jullian oscillation.

\section{Background}

The term monsoon is used to refer to the rainy phase of a seasonally changing pattern.In the Philippines, the Summer Monsoon (southwest) is called the Habagat and the Winter Monsoon (northeast) is called the Amihan. For Amihan weather pattern, it begins sometime in November or
December and ends sometime in May or June. There may, however, be wide variations from year to year. Throughout the rest of the year, the Philippines experiences the Habagat season.

Oscillations or fluctuations of rainfall are either in a form of a year-to-year (inter-annual) oscillations or decade-todecade (interdecadal) oscillations. Examples of interannual oscillations are the El Nino or La Nina phenomenon in the tropical Pacific. However, on sub-seasonal time scales, rainfall also fluctuates. These are referred to as intraseasonal oscillations (ISO). They appear as "active' and "break" spells of rainfall (Pillai and Aher, 2018). Low frequency ISOs are those with periodicity of 20-60 days. (Karmakar et al., 2015). The intraseasonal oscillations, and in particular the Madden Julian Oscillation (MJO) have been and still remain a "holy grail" of today's atmospheric science research. (Fuchs and Raymond, 2017). The MJO identified by Madden and Julian $(1971,1972)$ has been well recognized as the most prominent intraseasonal signal in the tropics. Its discovery and its relationship with other weather phenomena such as tropical cyclone (TC) are among the most significant advancements in modern meteorology with broad and far reaching impacts. The original study by Madden and Julian used radiosonde data on the Canton Island and their spectral analysis revealed the signal of a 40-50-day oscillation. (Li et al., 2018)

A recent research utilized Multichannel Singular Spectrum Analysis (MSSA) in analyzing the fluctuations of the intraseasonal oscillatory modes of India summer monsoon (Karmakar et al., 2015). MSSA is a natural extension of singular spectrum analysis (SSA) to a time series of vectors or maps, such as time-varying temperature or precipitation distributions over the globe. The spectral domain approach of singular spectrum analysis (SSA) is motivated by the observation that the most regular, and hence predictable, behavior of a time series is to be periodic. This approach then proceeds to determine the periodic components embedded in the time series by computing the associated periods, amplitudes, and phases. (Vautard and Ghil, 1989). 
Several studies has been done were MSSA was utilized to successfully identify and categorize spatio-temporal structures of ISO over the region in China (Fukutomi et al., 2012; Golyandina and Korobeynikov, 2012; Li et al., 2018) and North America (Mo and Mo, 2000). Similar study is not yet done in the Philippines summer monsoon, although a climatological analysis of the southwest monsoon in the Philippines was studied locally (Cruz et al., 2013), but it only looks at the inter-annual variability and the influence of the El Niño Southern Oscillation - hence this paper is motivated to do such.

Along with the inter-seasonal variability researches is the study on its association with extreme precipitation events. A study revealed that changes in extreme rainfall in the Philippines are linked to an inter-annual oscillation (Villafuerte et al., 2012). Again, there has been no study on its association with the intra-seasonal oscillation.

The overall objective of this study is to improve our understanding of the changing nature of the intra-seasonal oscillatory (ISO) modes of Philippine summer monsoon manifested by active and break phase, and their association with extreme rainfall events. The specific research objectives are:

1. Determine the time series of the total daily variance explained by the (a) low frequency intra-seasonal oscillations (LF-ISO) and (b) high frequency intra-seasonal oscillations (HF-ISO)

2. Determine the percentage ratio of the extreme events that occurred during the (a) negative (break) phase, (b) transition phase and (c) positive (active) phase of LF-ISO.

\section{Dataset}

This research will utilize the gridded daily rainfall dataset particularly the Daily Gridded CPC Precipitation for 1979 to present that were downloaded from CPC Global Unified Precipitation data provided by the NOAA/OAR/ESRL PSD, Boulder, Colorado, USA(https://www.esrl.noa.gov/psd/)T hese dataset is part of products suite from the CPC Unified Precipitation Project that are underway at NOAA Climate Prediction Center (CPC). Dataset is a PSD standard NetCDF about 65 Mbyte netCDF4 file for each yearly file. (Mo and Mo, 2000).

This endeavor also utilized the code of the package called Rssa for the implementation of the multivariate singular spectrum analysis. For the calculation of the four versions of Mann-Kendall Test, the package modifiedmk and HKprocess were used.

\section{MSSA decomposition}

The steps in carrying out multi-channel singular spectrum (MSSA) are analogous to a similar study done in India. (Karmakar et al., 2015). The climate of the country is divided into two main seasons: the rainy season, from June to October; the dry season, from November to May. The typhoon season is June to November, but occasionally typhoons occur outside of the typhoon season. But due to the arrivals of extreme events and typhoons during Decembers, this paper would like to analyze the data during the July-to-December period.
To extract oscillatory components present in the monsoon variability, MSSA is applied to the filtered June-December data ( $N=184$ days long) each year. Each year is treated separately because so that there would be no discontinuity in the rainfall time series. Additionally, since the characteristics of the intraseasonal variability vary on a year-to-year basis, it makes sense to examine each year individually. The embedding window length $(\mathrm{M})$ is taken as 60.

Usually, an oscillation is identifiable if eigenvalues associated with two RCs are nearly equal and corresponding eigenvectors represent almost equal dominant frequency with phase quadrature. The sum of these individual pair of PC represents the oscillation.

The amplitude and the phase angle of the oscillation can be determined using the first principal component (PC) of a particular oscillatory component.

\subsection{Extracting ISO modes and defining active/break phases}

The ISO modes are captured by applying multichannel singular spectrum analysis (MSSA) to the June-December (184 days long) data over Philippines for each year. This separates out oscillatory modes from noisy data, following which we determine the spatial-temporal structures associated with those characterized by that part of the spectrum.

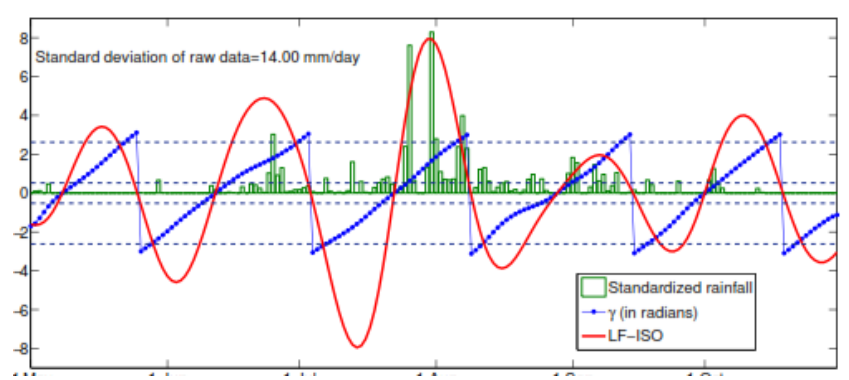

Figure 1. Defining the phases of LF-ISO at a particular gridpoint $(122.75 \mathrm{E}, 10.75 \mathrm{~N})$ in 2006 . Units are in $\mathrm{mm} /$ day. The phase angle is shown by blue dots (in radians). Adapted from

"Decreasing intensity of monsoon low-frequency intraseasonal variability over India." Karmakar, N., Chakraborty, A., \& Nanjundiah, R.S. (2015) Environmental Research Letters, 10(5). https://doi.org/10.1088/1748-9326/10/5/054018

Since the length of the lag window is 60 days, each MSSA mode consists of an ST-EOF which is a sequence of 60 lagged maps. The ST-EOF and ST-PC are used to construct the space-time RC of each eigenmode (see (Vautard and Ghil, 1989). The RCs are on the same spatial grid and have the same time length and sequence as the original data.

In order to determine the phase of an ISO cycle at a particular gridpoint, we use the following equation:

$$
\gamma(\mathrm{t})=\operatorname{Arg}\left(\mathrm{Y}^{\prime}(\mathrm{t})+\mathrm{iY}(\mathrm{t})\right)
$$

where, $\mathrm{t}$ denotes time, $\mathrm{Y}(\mathrm{t})$ is the 184 day long (JuneDecember) ISO (LF-ISO or HF-ISO) time series at any point and indicates the time-derivative. $\operatorname{Arg}(z)$ represents the principal value of the phase of a complex number $\mathrm{z}=\mathrm{x}+$ 
iy. $\gamma(\mathrm{t})$ is calculated at every gridpoint each year and lies between $-\pi$ and $\pi$. (Karmakar et al., 2015) Active (positive), break (negative) and transition phases of ISO are defined based on the values of $\gamma(t)$.

Table 1. The Periodicities and \% Varianceof theLF-ISO and HF-ISO

\begin{tabular}{|c|c|c|c|c|c|c|}
\hline \multirow{2}{*}{ Year } & \multicolumn{3}{|c|}{ Low-Frequency ISO (20-60) } & \multicolumn{3}{|c|}{ High-Frequency ISO (10-20) } \\
\hline & Mode & Periodicity & \%Variance & Mode & Periodicity & \%Variance \\
\hline 1979 & 2,3 & 53.8 & 8.52 & 4,5 & 19.3 & 5.86 \\
\hline 1980 & 2,3 & 57.7 & 6.74 & 6,7 & 19.4 & 4.55 \\
\hline 1981 & 2,3 & 37.3 & 6.53 & 6,7 & 14.9 & 5.11 \\
\hline 1982 & 4,5 & 21.2 & 5.68 & 2,3 & 19.9 & 6.05 \\
\hline 1983 & 3,4 & 43.8 & 6.94 & 5,6 & 17.7 & 5.56 \\
\hline 1984 & 12,13 & 28.9 & 4.32 & 4,5 & 18.7 & 6.7 \\
\hline 1985 & 7,8 & 29.2 & 4.38 & 3,4 & 13.2 & 6.96 \\
\hline 1986 & 2,3 & 27.4 & 19.75 & 9,10 & 11.2 & 4.24 \\
\hline 1987 & 5,6 & 26.8 & 4.27 & 6,7 & 10.1 & 4 \\
\hline 1988 & 12,13 & 42.5 & 3.25 & 6,7 & 11.6 & 3.95 \\
\hline 1989 & 2,3 & 31.1 & 6.66 & 8,9 & 18.9 & 3.85 \\
\hline 1990 & 3,4 & 57.7 & 5.7 & 6,7 & 14.8 & 3.75 \\
\hline 1991 & 4,5 & 44.1 & 4.42 & 3,4 & 12.9 & 4.66 \\
\hline 1992 & 3,4 & 41.3 & 5.35 & 5,6 & 19.7 & 6.91 \\
\hline 1993 & 2,3 & 34.9 & 6.0 & 3,4 & 14.9 & 5.88 \\
\hline 1994 & 3,4 & 54.5 & 6.01 & 4,5 & 15.1 & 4.72 \\
\hline 1995 & 5,6 & 24.4 & 4.37 & 7,8 & 17.5 & 3.75 \\
\hline 1996 & 2,3 & 48.2 & 7.0 & 7,8 & 10.17 & 4.29 \\
\hline 1997 & 4,5 & 49.2 & 5.91 & 3,4 & 12.2 & 6.34 \\
\hline 1998 & 2,3 & 42.6 & 6.82 & 3,4 & 13.6 & 6.48 \\
\hline 1999 & 9,10 & 30.6 & 4.24 & 3,4 & 15.3 & 5.32 \\
\hline 2000 & 2,3 & 29.4 & 5.99 & 10,11 & 10.8 & 3.76 \\
\hline 2001 & 2,3 & 49.5 & 5.7 & 3,4 & 14.3 & 5.38 \\
\hline 2002 & 2,3 & 25.6 & 8.39 & 3,4 & 19.2 & 6.47 \\
\hline 2003 & 3,4 & 37.4 & 6.01 & 2,3 & 14.7 & 5.41 \\
\hline 2004 & 5,6 & 29.5 & 8.23 & 2,3 & 15.7 & 5.31 \\
\hline 2005 & 2,3 & 22.1 & 5.52 & 8,9 & 10.83 & 6.57 \\
\hline 2006 & 5,6 & 36.5 & 2.57 & 2,3 & 11.2 & 3.12 \\
\hline 2007 & 2,3 & 52.5 & 7.77 & 7,8 & 16.2 & 4.92 \\
\hline 2008 & 4,5 & 44.7 & 5.0 & 2,3 & 12.2 & 6.67 \\
\hline 2009 & 2,3 & 41.6 & 6.88 & 2,3 & 14.1 & 5.73 \\
\hline 2010 & 2,3 & 25.7 & 6.06 & 8,9 & 17.9 & 4.29 \\
\hline 2011 & 2,3 & 29.5 & 8.65 & 4,5 & 17.1 & 5.44 \\
\hline 2012 & 11,12 & 24.1 & 3.37 & 4,5 & 16.6 & 4.41 \\
\hline 2013 & 3,4 & 33.2 & 6.81 & 11,12 & 13.6 & 3.05 \\
\hline 2014 & 4,5 & 35.23 & 5.94 & 8,9 & 10.3 & 4.51 \\
\hline 2015 & 2,3 & 37.2 & 6.45 & 6,7 & 16.4 & 4.84 \\
\hline 2016 & 3,4 & 27.3 & 5.83 & 6,7 & 10.2 & 4.45 \\
\hline 2017 & 2,3 & 52.9 & 7.77 & 7,8 & 16.2 & 4.92 \\
\hline
\end{tabular}

For illustration, in Figure $1, \gamma(\mathrm{t})$ is calculated for LF-ISO at a gridpoint in central India for May-October 1951. The active, break and transition phases are defined in the following way: If for a value of $t, \gamma(t)$ lies within $\pi / 6$ and $5 \pi / 6$, ISO is in active (positive) phase, and if $\gamma(\mathrm{t})$ lies within $-5 \pi / 6$ and $-\pi / 6$, ISO is in break (negative) phase. The rest of the values of $\gamma(\mathrm{t})$ determines the transition phase.

Karmakar used the classical Mann-Kendall non-parametric testto identify the significant trends. In the study of Dinpashoh (Dinpashoh et al., 2014) and Zamani (Zamani et al., 2016) four different versions of Mann-Kendall were used. This paper utilized the classic Mann-Kendall (MK1); modified Mann-Kendall which detrends data using lag-1 autocorrelation (MK2); modified Mann-Kendall which serial correlation coefficients for all lags are used (MK3); and Mann-Kendall methods that make inference about the Hurst-Kolmogorov and the AR(1) process (MK4). Associated slopes are estimated using the median of all pairwise slopes in the data set (Theil-Sen estimator).

\subsection{Extreme events}

Extreme precipitation events are episodes of abnormally high rainfall. One definition of an extreme precipitation event is the persistent extreme precipitation event (PEPE) based on precipitation data at individual meteorological stations. It requires daily precipitation exceeding the threshold of $50 \mathrm{~mm}$ and those persisting for at least three 
consecutive days at each individual observational station. (Chen and Zhai, 2013). In this study, another definition is considered which follows the work of Karmakar. (Karmakar et al., 2015) The thresholds for extreme events are those 99.5th percentile values (results are insensitive to small changes in percentile threshold) at each of the gridded data pointbased on July-December data for 1979-2016. So, each location has a different threshold for extreme rainfall.

\section{Results}

\subsection{The pattern of LF-ISO and HF-ISO}

The time series in Figure 2 shows the cumulative variance explained by the LF-ISO associated with the reconstructed MSSA output of the first significant eigenmode pair for the time period 1979-2016.

The eigenvalues obtained from MSSA represent the power in the extracted modes. When we apply MSSA to rainfall data, several oscillatory modes, occurring usually in pairs, are detected every year, and are significantly different from those generated by pure noise. The sums of the variances explained by the significant eigenmodes in lowand high-frequency scales represent the intensities of LFISO and HF-ISO each year relative to the total power in the intraseasonal band, and we define the respective sums as the LF-ISO and HF-ISO indices.(Karmakar et al., 2015).

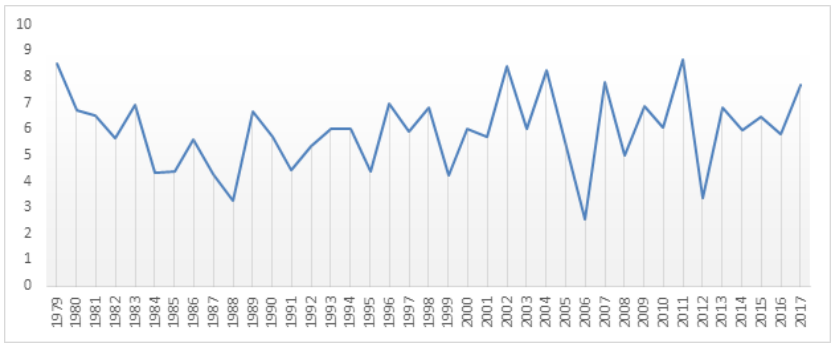

Figure 2. Time series of the cumulative variance explained by the significant eigenmodes (in percentage) of the intraseasonal band (8-90) days that represents low- frequency oscillatory behavior with periodicities of 20-60 days

Investigating for the trend in the LF-ISO, Mann Kendall test showed that there was no significant trend in the LF-ISO for the time period 1979-2016 (Tau $=0.104,2$-sided pvalue $=$ 0.357). However, visually looking at the graph (Figure 2), a slowly increasing trend emerges if one considers the time period 1984-2016. Mann Kendall test (Tau $=0.28,2$-sided pvalue $=0.0207$ ) suggests a significant trend and the TheilSen estimator provided a slope of 0.05 during this timeperiod. When the classic MK1 is significant, all the other MK tests are also significant.

The time series in Figure 3. shows the cumulative variance explained by the HF-ISO associated with the reconstructed MSSA output of the first significant eigenmode pairs for the time period 1979-2016.

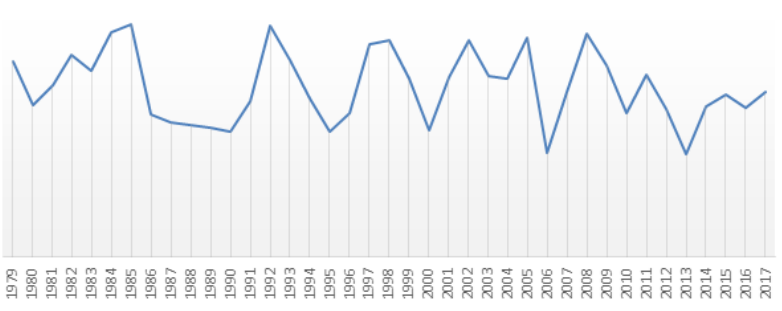

Figure 3. Time series of the cumulative variance explained by the significant eigenmodes (in percentage) of the intraseasonal band (8-90) days that represents high-frequency oscillatory behavior with periodicities of 10-20 days

\subsection{Active and break phases}

After summer, at the onset of July comes the rainy season in the Philippines. The start of rainfall variability during July can be characterized by the first eigenvector. This trend indicates the dominant variation in the data occurs over space.

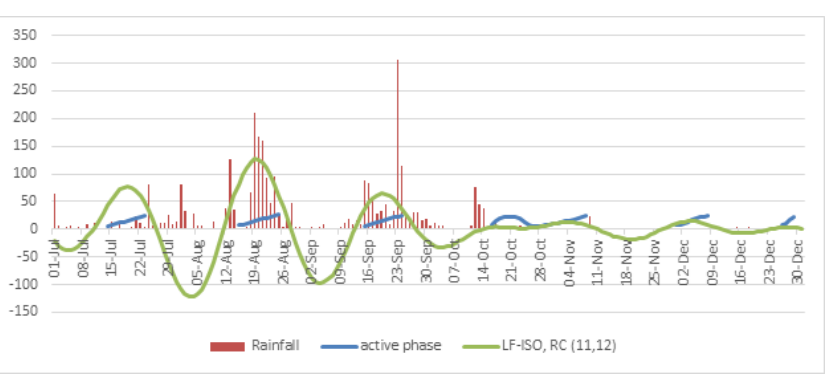

Figure 4. Defining the phases of LF-ISO at a particular location (CUBI Station, Lat 14.75, Lon 120.25) in 2012. Green curve indicates the LF-ISO and red boxes are the rainfall at that location. Units are in $\mathrm{mm} /$ day. The blue dotted line indicates the active phase of the LF-ISO obtained from RC $(11,12)$

Based on the oscillatory behavior of the reconstructed ISOs, we define the active (positive), break (negative) and transition phases using the ISO time series (LF-ISO or HFISO). This classification, therefore, purely depends upon the nature of the eigenmodes determined by MSSA. Typically, there are 4-6 LF-ISO cycles in one July-December season. In this study, we only utilized the first mode associated with a LF-ISO and HF-ISO.

The LF-ISO cycle obtained from RC $(11,12)$ is superimposed in the graph of the standardized daily rainfall at a particular grid (CUBI Station, lat 14.75, lon 120.25). See Figure 4.

The phenomena behind these intraseasonal oscillation is strongly related to the Madden Julian Oscillation. Tropical cyclone frequency has strong variations on both the intraseasonal and longer timescales. On the intraseasonal timescale, tropical cyclone formation is strongly modulated by the Madden-Julian Oscillation (MJO). (North, Pyle, \& Zhang, n.d.). The MJO was first discovered in the early 1970s by Dr. Roland Madden and Dr. Paul Julian when they were studying tropical wind and pressure patterns. They often noticed regular oscillations in winds (as defined from departures from average) between Singapore and Canton 
Island in the west central equatorial Pacific. (Madden, Julian, Madden, \& Julian, 1971).

Table 2. Extreme events happening during LF-ISO and HF-ISO

\begin{tabular}{|c|c|c|c|}
\hline \multirow{2}{*}{ Year } & \multicolumn{3}{|c|}{ Percentage Ratio of Extreme Events During LF-ISO } \\
\hline & Active Phase & Break Phase & Transition Phase \\
\hline 1984 & 40.37 & 27.81 & 31.82 \\
\hline 1985 & 18.75 & 58.75 & 22.50 \\
\hline 1986 & 33.80 & 44.09 & 22.11 \\
\hline 1987 & 27.33 & 52.17 & 20.50 \\
\hline 1988 & 48.75 & 35.63 & 15.63 \\
\hline 1989 & 38.13 & 24.38 & 37.50 \\
\hline 1990 & 27.50 & 46.25 & 26.25 \\
\hline 1991 & 21.88 & 45.00 & 33.13 \\
\hline 1992 & 35.63 & 37.92 & 26.46 \\
\hline 1993 & 21.25 & 61.88 & 16.88 \\
\hline 1994 & 52.50 & 25.00 & 22.50 \\
\hline 1995 & 52.50 & 25.00 & 22.50 \\
\hline 1996 & 58.39 & 22.36 & 19.25 \\
\hline 1997 & 23.75 & 57.50 & 18.75 \\
\hline 1998 & 47.50 & 30.00 & 22.50 \\
\hline 1999 & 18.75 & 56.25 & 25.00 \\
\hline 2000 & 37.27 & 37.89 & 24.84 \\
\hline 2001 & 35.10 & 41.19 & 23.71 \\
\hline 2002 & 36.88 & 40.63 & 22.50 \\
\hline 2003 & 31.71 & 46.51 & 21.78 \\
\hline 2004 & 36.13 & 42.44 & 21.44 \\
\hline 2005 & 33.95 & 48.77 & 17.28 \\
\hline 2006 & 18.75 & 58.75 & 22.50 \\
\hline 2007 & 54.94 & 21.60 & 23.46 \\
\hline 2008 & 43.89 & 35.21 & 20.90 \\
\hline 2009 & 50.00 & 31.48 & 18.52 \\
\hline 2010 & 51.85 & 29.01 & 19.14 \\
\hline 2011 & 25.63 & 53.13 & 21.25 \\
\hline 2012 & 50.63 & 16.88 & 32.50 \\
\hline 2013 & 51.55 & 16.15 & 32.30 \\
\hline 2014 & 43.14 & 38.28 & 51.91 \\
\hline 2015 & 21.12 & 36.02 & 42.86 \\
\hline 2016 & 49.28 & 84.06 & 32.29 \\
\hline 2017 & 18.75 & 56.88 & 24.38 \\
\hline Average & 36.98 & 40.73 & 22.28 \\
\hline
\end{tabular}

Table 3. Trend analysis using the four Mann-Kendall tests

\begin{tabular}{ccccccccc}
\hline & \multicolumn{2}{c}{ MK1 } & \multicolumn{2}{c}{ MK2 } & \multicolumn{2}{c}{ MK3 } & \multicolumn{1}{c}{ MK4 } \\
\cline { 2 - 10 } & Tau & p-value & Tau & p-value & Tau & p-value & Tau & p-value \\
\hline Active Phase & 0.0789 & 0.522 & 0.0789 & 0.522 & 0.0789 & $\mathbf{0 . 0 1 6}$ & 0.0789 & 0.523 \\
\hline Break Phase & -0.141 & 0.247 & -0.141 & 0.247 & -0.141 & 0.247 & -0.141 & 0.247 \\
\hline Transition Phase & 0.111 & 0.367 & 0.111 & 0.506 & 0.111 & 0.282 & 0.111 & 0.368 \\
\hline
\end{tabular}

The MJO consists of two parts, or phases: one is the enhanced rainfall (or convective) phase and the other is the suppressed rainfall phase. Strong MJO activity often dissects the planet into halves: one half within the enhanced convective phase and the other half in the suppressed convective phase. These two phases produce opposite changes in clouds and rainfall and this entire dipole (i.e., having two main opposing centers of action) propagates eastward.

\subsection{Association with extreme events}

In order to understand how the occurrence of extreme events is associated with LF-ISO, we looked into the active phase and break phase of an LF-ISO and the number of extreme events occurring in that particular phase.

We tracked down the number of occurrence of an extreme event, being defined as the $95^{\text {th }}$ percentile of every rainfall data in a specific location every year. For example, the percentage ratio of extreme events that occurred in the 
active, break and transition phase during 1984are 40.37\%, $27.81 \%$ an $31.82 \%$ (see Table 2 ).

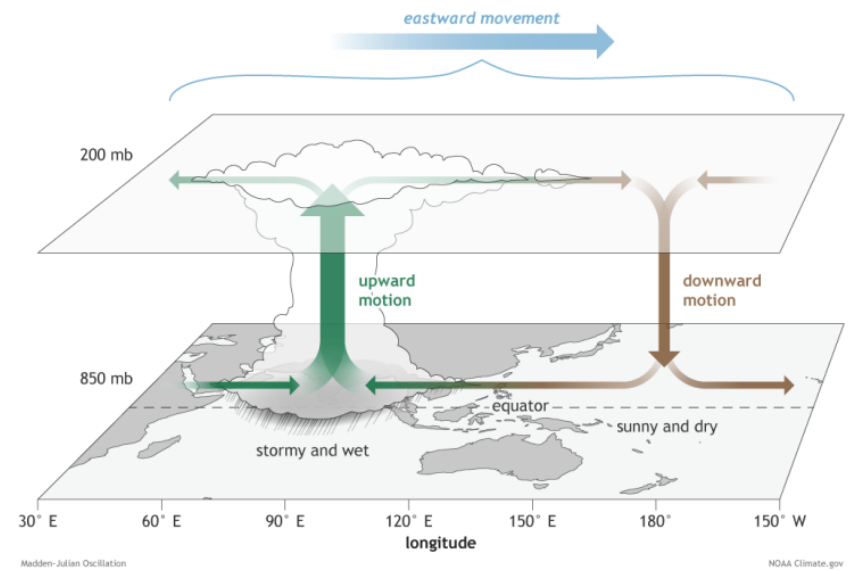

Figure 5. The surface and upper-atmosphere structure of the MJO for a period when the enhanced convective phase (thunderstorm cloud) is centered across the Indian Ocean and the suppressed convective phase is centered over the westcentral Pacific Ocean. Horizontal arrows pointing left represent wind departures from average that are easterly, and arrows pointing right represent wind departures from average that are westerly. The entire system shifts eastward over time, eventually circling the globe and returning to its point of origin. Reprinted from What is the MJO, from https://www.climate.gov/news-

features/blogs/enso/what-mjo-and-why-do-we-care

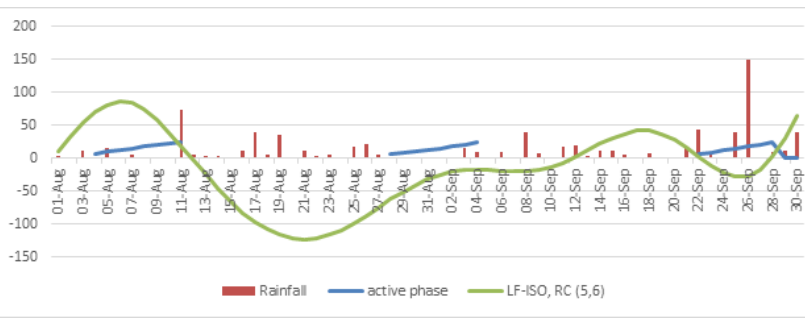

Figure 6. The phases of LF-ISO at a particular location (Laoag Station) in 2012. The high volume of rainfall in August 7, 2009 clearly falls in the active phase of the LF-ISO (blue dotted line)

Rappler (2012) reported in their website that the accumulated rainfall in August 7, 2009 is higher compared accumulated rainfall during tropical storm Ondoy (international codename Ketsana) back in Sept 26, 2009 measured at the Pagasa Science Garden in Quezon City.Looking at Figure 6, this extreme rainfall event resides within the active phase of the LF-ISO.

The time series of the percentage ratio occurrence of extreme events during the active, break and transition phases is shown in Figure 7.

During the active stages, there is a slight positive trend as provided by the Theil-Sen estimator (slope $=0.21$ ). However this is not a significant (increasing) trend as provided by the $p$ values of the three versions of Mann Kendall test; the MK1, MK2 and MK4 as shown in Table 3. Take note that the four tests have similar Tau values (Tau $=0.0789$ ). MK3 test, on the other hand, implies that this increasing trend is significant (pvalue $=0.016$ ). This implies that if we consider the serial correlation coefficients for all lags in calculating the effective sample size to be used in correcting the variance - then the trend is believed to be significant.

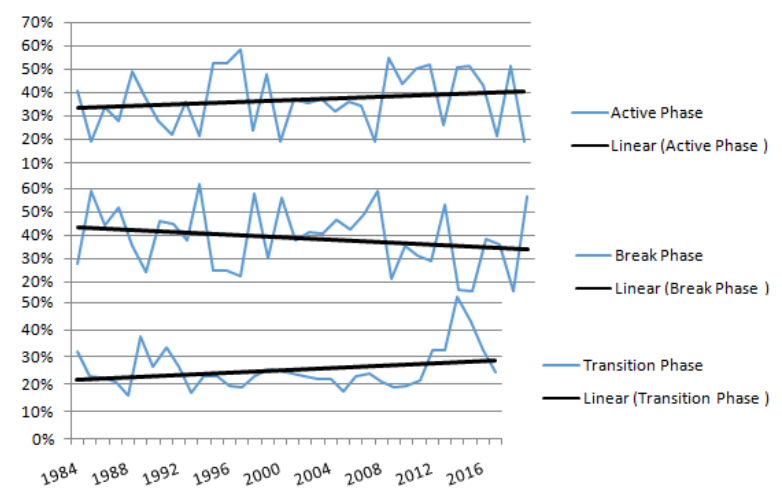

Figure 7. Time series (from 1985-2016) of the percentage ratio of the extreme events that occurred in the active phase, break phase and transition phase of LF-ISO, to the total number of extreme rainfall events each year

The modified Mann-Kendall tests MK2 and MK3 both addressed the issue of a serially correlation data - and gave two opposing claims that one is significant and other is not. But the main concern here is that there is an increasing trend in the occurrence of increased rainfall during the active stage.

During the break phase, Mann Kendall test were Tau $=-0.141,2$-sided pvalue $=0.247$ and the Theil-Sen estimator gave the slope -0.31 . There is decreasing trend during the break phase, however this trend is not significant. The occurrence of extreme events during a transition phase remains stable having a Theil-Sen estimate of its slope at 0.043 .

Overall, extreme precipitation events occurred mostly during the break phase of an LF-ISO. The average percentage ratio from the year 1984 to 2016 is $40.73 \%$ during the break phase compared to $36.98 \%$ during the active phase. This in consonance with the study of Karmakar (2012) where there is an increasing percentage of extreme events happening during the break phase and transition phase; and decreasing trend during the active phase.

\section{Conclusions and recommendation}

We have studied the intraseasonal low-frequency oscillation using gridded daily rainfall dataset particularly the Daily Gridded CPC Precipitation for 1979 to present. Multivariate Singular Spectrum Analysis (SSA) was used in the extraction of these modes. We have found out that there is a significant increasing trend in the relative strength of low frequency ISO. However, this happened only after the years 1979 to 1984 was removed in the trend analysis. It is suggested that an in-depth look on these years before 1985 should be taken into investigation in order to shed light on the decreasing trend during this period. 
The significant increasing trend of the LF-ISO is not in tune with the decreasing trend (slope $=0.02$ ) of the LF-ISO in India. This might be because of the phenomenon called Madden-Julian Oscillation (MJO). Recent studies have been conducted and established the influence of MJO on the intraseasonal precipitation variability over India (Pai, Bhate, Sreejith, \& Hatwar, 2011), Southern Iran (Pourasghar et al., 2015) and northeast Brazil (Valadão, Carvalho, Lucio, \& Chaves, 2017). It is suggested that an investigation of the influence of MJO in the vicinity of the Philippines should be done.

The increased rainfall during the break and transition phase of LF-ISO (in the form of extreme events) could be a contributing factor to the reduction in LF-ISO intensity through changes in its amplitude and variability (Karmakar et al., 2015). In this study, the implication is rather the opposite. This means that the increase in rainfall during the active phase of LF-ISO could be a contributor to the decreasing trend of rainfall during the break phase. This is in accord with the Madden-Jullian Oscillation, where there is a decreasing trend at the first stage of MJO (somewhere in India) and eventually moving eastward resulting to an increasing trend at the second stage of MJO (somewhere in the Philippines). Karmakar also noted the existence of multi-decadal shifts that influenced the increase of the variability of LF- ISO. It is suggested that multi-decadal shifts should be done, although it will take more than 100 years of data in order to carry out the study.

Relative to extreme precipitation, a recent study (Sun, Jiang, Ren, \& Yang, 2016) suggested that proper combination of different intraseasonal oscillations could effectively predict the persistent extreme precipitation event. The study found out that North Indian Ocean is the major forcing region of 30-60 day ISO, where anomalous warm sea surface temperature-induced local strong convections stimulate a meridional teleconnection wave train over the East Asia, generating the 30-60 day intraseasonal rainfalls. The 10-30 day ISO primarily originates from the northwest Pacific and the South China Sea (SCS), and along with its northwestward and northeastward propagations due to the air-sea coupling and prevailing winds, suppressed and enhanced convections appear alternatively over the Philippine Sea and the central SCS. These are all in synchronization with the Madden Julian oscillation.

Overall, it is intriguing to know the factors that may have played a role in increasing intensity of LF-ISO. But there is no accord yet on how the ISO modes will behave in a changing climate, and it remains a very difficult problem to be addressed, as the state-of-the-art models still have difficulties in simulating the mean picture of ISO (Karmakar et al., 2015).

\section{References}

Chen Y. and Zhai P. (2013), Persistent extreme precipitation events in China during 1951-2010, Climate Research, 57(2), 143-155. https://doi.org/10.3354/cr01171.

Cruz F.T., Narisma G.T., Villafuerte M.Q., Cheng Chua K.U. and Olaguera L.M. (2013), A climatological analysis of the southwest monsoon rainfall in the Philippines, Atmospheric
Research, 122, 609-616. https://doi.org/10.1016/j.atmosres. 2012.06.010.

Dispanhoh Y., Mirabbasi R., Jhajharia D., Abianeh H. and Mostafaeipour A. (2014), Effect of short-term and long-term persistence on identification of temporal trends, Journal of Hydrologic Engineering, 19(3). https://doi/10.1061/\%28 ASCE\%29HE.1943-5584.0000819.

Fuchs Ž. and Raymond D.J. (2017), A simple model of intraseasonal oscillations, Journal of Advances in Modeling Earth Systems, 9(2), 1195-1211. https://doi.org/10.1002/ 2017MS000963.

Fukutomi Y., Masuda K. and Yasunari T. (2012), Spatiotemporal structures of the intraseasonal oscillations of precipitation over northern Eurasia during summer, International Journal of Climatology, 32(5), 710-726. https://doi.org/10.1002/ joc.2293.

Golyandina N. and Korobeynikov A. (2012), Basic Singular Spectrum Analysis and Forecasting with $\mathrm{R}$, https://doi.org/10.1016/j.csda.2013.04.009.

Golyandina N., Korobeynikov A., Shlemov A. and Usevich K. (2013), Multivariate and 2D Extensions of Singular Spectrum Analysis with the Rssa Package, https://doi.org/10.18637/jss.v067.i02.

Karmakar N., Chakraborty A. and Nanjundiah R. S. (2015), Decreasing intensity of monsoon low-frequency intraseasonal variability over India, Environmental Research Letters, 10(5), https://doi.org/10.1088/1748-9326/10/5/054018.

Li T., Wang L., Peng M., Wang B., Zhang C., Lau W., ... Kuo H.-C. (2018), A Paper on the Tropical Intraseasonal Oscillation Published in 1963 in a Chinese Journal, Bulletin of the American Meteorological Society, BAMS-D-17-0216.1. https://doi.org/10.1175/BAMS-D-17-0216.1.

Madden R.A., Julian P.R., Madden R.A. and Julian P.R. (1971), Detection of a 40-50 Day Oscillation in the Zonal Wind in the Tropical Pacific, Journal of the Atmospheric Sciences, 28(5), 702-708. https://doi.org/10.1175/1520-0469(1971)028<070 2:DOADOI>2.0.CO;2.

Mo K.C. and Mo K.C. (2000), Intraseasonal Modulation of Summer Precipitation over North America, Monthly Weather Review, 128(5), 1490-1505. https://doi.org/10.1175/15200493(2000)128<1490:IMOSPO>2.0.CO;2.

North G.R. and Pyle J.C. (John C. and Zhang F. (n.d.). Encyclopedia of atmospheric sciences. Retrieved from https://www.sciencedirect.com/referencework/9780123822 253/encyclopedia-of-atmospheric-sciences.

Pai D.S., Bhate J., Sreejith O.P. and Hatwar H.R. (2011), Impact of MJO on the intraseasonal variation of summer monsoon rainfall over India, Climate Dynamics, 36(1-2), 41-55. https://doi.org/10.1007/s00382-009-0634-4.

Pillai P.A. and Aher V.R. (2018), Role of monsoon intraseasonal oscillation and its interannual variability in simulation of seasonal mean in CFSv2, Theoretical and Applied Climatology, 131(1-2), 745-760. https://doi.org/10.1007/ s00704-016-2006-4.

Pourasghar F., Tozuka T., Ghaemi H., Oettli P., Jahanbakhsh S. and Yamagata T. (2015), Influences of the MJO on intraseasonal rainfall variability over southern Iran, Atmospheric Science Letters, 16(2), 110-118. https://doi.org/10.1002/as/2.531.

Sun X., Jiang G., Ren X. and Yang X.-Q. (2016), Role of intraseasonal oscillation in the persistent extreme precipitation over the Yangtze River Basin during June 1998, 
Journal of Geophysical Research: Atmospheres, 121(18), 10453-10469. https://doi.org/10.1002/2016JD025077.

Valadão C.E.A., Carvalho L.M.V., Lucio P.S. and Chaves R.R. (2017), Impacts of the Madden-Julian oscillation on intraseasonal precipitation over Northeast Brazil, International Journal of Climatology, 37(4), 1859-1884. https://doi.org/10.1002/joc.4818.

Vautard R. and Ghil M. (1989), Singular spectrum analysis in nonlinear dynamics, with applications to paleoclimatic time series. Physica D: Nonlinear Phenomena, 35(3), 395-424. https://doi.org/10.1016/0167-2789(89)90077-8.

Zamani R., Mirabbasi R., Abdollahi S. and Jhajharia D. (2017), Streamflow trend analysis by considering autocorrelation structure, long persistence, and Hurst coefficient in a semiarid region of Iran. Theoretical and Applied Climatology, 129, 33-45. https://doi.org/10.1007/s00704-016-1747-4. 